\title{
HyperLEDA. III. The catalogue of extragalactic distances
}

\author{
Dmitry Makarov ${ }^{1,2}$, Philippe Prugniel $^{1}$, Nataliya Terekhova $^{3}$, Hélène Courtois $^{4}$, and Isabelle Vauglin ${ }^{1}$ \\ 1 Université Lyon 1, Villeurbanne, 69622, France; CRAL, Observatoire de Lyon, St. Genis Laval, 69561, France; CNRS UMR 5574, \\ France \\ e-mail: dim@sao.ru \\ 2 Special Astrophysical Observatory, Nizhniy Arkhyz, 369167 Karachai-Cherkessia, Russia \\ 3 Sternberg Astronomical Institute, Moscow State University, Universitetsky pr., 13, 119991 Moscow, Russia \\ ${ }^{4}$ Université Claude Bernard Lyon I, Institut de Physique Nucléaire, 69622 Lyon, France
}

Received 23 January 2014 / Accepted 6 August 2014

\begin{abstract}
We present the compilation catalogue of redshift-independent distances included in the HyperLEDA database. It is actively maintained to be up-to-date, and the current version counts 6640 distance measurements for 2335 galaxies compiled from 430 published articles. Each individual series is recalibrated onto a common distance scale based on a carefully selected set of high-quality measurements. This information together with data on $\mathrm{H}$ I line width, central velocity dispersion, magnitudes, diameters, and redshift is used to derive a homogeneous distance estimate and physical properties of galaxies, such as their absolute magnitudes and intrinsic size.
\end{abstract}

Key words. astronomical databases: miscellaneous - catalogs - galaxies: distances and redshifts

\section{Introduction}

Measuring distances is a long-standing and on-going goal of astronomy. Significant milestones were the measurement of the distance to the Moon by Hipparchus (about $150 \mathrm{BC}$, discussed by Toomer 1974), and, in the modern era, the determination of the Cepheid distances, which unveiled the true nature of galaxies (Hubble 1926).

Although the discovery of a relation between the distance and the radial velocity of galaxies (Hubble 1929) built the paradigm of an expanding Universe and gave a simple "proxy" to the evaluation of extragalactic distances, redshift-independent distances are still vital. In particular, the deviation of radial velocities from the Hubble law gives us information about cosmic flows (Tully et al. 2008) and mass distributions (Karachentsev et al. 2009; Courtois et al. 2012). The distances are also obviously needed to fix the Hubble constant, $H_{0}$, and they are crucial to constrain the cosmological parameters. Most notably, the use of distant supernovae as standard candles led to the discovery of the accelerated expansion of the Universe (Riess et al. 1998; Perlmutter et al. 1999). These observations together with other evidence form the modern standard cosmological model, according to which our Universe is mostly "dark". It consists of about $73 \%$ of dark energy, $22 \%$ dark matter, and only $\sim 5 \%$ of baryon matter (Jarosik et al. 2011).

There are many indicators from which extragalactic distances can be derived. The best-calibrated and most precise methods are usually observationally expensive and limited to the nearby Universe, for example the period-luminosity relation for Cepheids. At larger distance, most indicators are based on scaling laws of galaxies, for example, the well-known Tully-Fisher relation. Because no single technique can work on all scales, a consistent system is constructed step-by-step, from nearby to distant objects. In this process, each distance indicator is calibrated with respect to those available at nearest scales. For this purpose it is necessary to maintain a database of precise measurements that can be homogenized into a common scale that can be used as standards.

The goal of the present work is to describe the galaxy distance catalogue maintained within the HyperLEDA database ${ }^{1}$. It compiles distances published in the astronomical literature and provides precise descriptions of these measurements. In addition to this catalogue, HyperLEDA contains a consistent body of data from the literature on photometry, $\mathrm{H}$ I line width, internal stellar kinematics, and other characteristics. All these catalogues are combined and are corrected for systematic effects to provide a homogenized description of galaxies. In particular, the distances to galaxies are derived using the present catalogue supplemented by distance indicators based on other data (Tully-Fisher, Faber-Jackson, and Fundamental Plane relations).

HyperLEDA, including the distance catalogue, is also used for other projects. One of them is the Catalogue of the Local Volume galaxies $^{2}$ (LVG; Karachentsev et al. 2004, 2013), which gives distances, velocities, and physical characteristics for galaxies within $11 \mathrm{Mpc}$ (at present 869 galaxies). The LVG results from a detailed and careful analysis of the data collected in databases and in the literature. Special attention is given to cleaning the data from artefacts, Galactic objects, and doubtful measurements. This makes LVG much more than a mere subsample extracted from general databases. Ongoing efforts make LVG the most complete sample of nearby galaxies, that is virtually free of contamination.

Another project using the present catalogue is the Extragalactic Distance Database ${ }^{3}$ (Tully et al. 2009, EDD). It is intended to collect information related to the distance determination within 100-200 Mpc. EDD combines original observations with published data and compilations. Its Tully-Fisher relation is derived from a uniform analysis of the $\mathrm{H}$ I line width

\footnotetext{
http://leda.univ-lyon1.fr/

2 http://www.sao.ru/lv/lvgdb/

http://edd.ifa.hawaii.edu/
} 
(Courtois et al. 2009) and photometry (Courtois et al. 2011), whereas the zero-point calibration is derived from original distance determination using the colour-magnitude diagrams of nearby galaxies from the Hubble Space Telescope (Jacobs et al. 2009). The calibration sample for short distances is supplemented by the present catalogue.

The NASA/IPAC Extragalactic Database ${ }^{4}$ (NED) maintains an on-line compilation of redshift-independent extragalactic distances from the literature (NED-D). It collects huge sets of published measurements based on both primary methods such as Cepheids or Type Ia supernova (SN Ia), and secondary indicators such as Tully-Fisher or Fundamental Plane relations.

The present database is more focused on the nearby galaxies to provide the best standards to calibrate distance indicators. In Sect. 2 we briefly describe the organization and content of HyperLEDA, Sect. 3 describes the distance catalogue, Sect. 4 focuses on the homogenization of different distance determination methods, and Sect. 5 draws conclusions.

\section{HyperLEDA database}

HyperLEDA (Paturel et al. 2003a,b) takes its roots in the LyonMeudon Extragalactic database (Paturel et al. 1988, LEDA), which was created in 1983, and in Hypercat, which started as the observational catalogue on kinematics of early-type galaxies (Prugniel \& Simien 1996). These databases were joined in 2000. Historically, the LEDA database was used for preparation of the Third Reference Catalogue of Bright Galaxies (de Vaucouleurs et al. 1991, RC3). Now HyperLEDA is operated by the Observatoire de Lyon (France) and by the Special Astrophysical Observatory (Russia).

The basis of HyperLEDA is a set of compilation catalogues that are maintained on a regular basis. Each of them collects specific data on astronomical objects from the literature. The maintenance of these catalogues involves efforts to provide accurate cross-identifications of the celestial sources, a clear description of each series of measurements (including a documentation on the precision and systematic errors), and a flagging of doubtful or erroneous data.

The main part, the so-called LEDA catalogue, combines information from the compilations to provide a uniform and selfconsistent description of all objects. It consists of homogenized observations such as the total apparent magnitude in the $B$ band, apparent diameter, and redshift, as well as physical parameters including the absolute magnitude and the maximum rotation velocity. The homogenization process is based on the description of each series of measurements (for example how to apply an aperture correction) and on a statistical comparison of these series over the whole catalogue (for example how to correct zero-points). The physical values are determined from the homogenized apparent data, taking into account different kinds of corrections (Galactic extinction, object inclination, line-width broadening because of redshift, and others). The homogenization and parameter determination are described by Paturel et al. (1997). More recently, some aspects where revised by Paturel et al. (2003a,b). The online documentation provides updated details.

The principal compilation catalogues of HyperLEDA are described below.

Astrometry and Designation: these catalogues contain
8177892 celestial positions and 6878482 designa-
tions for 3730169 objects. The designations stored in the

4 http://ned.ipac.caltech.edu/ database comply with the IAU recommendation ${ }^{5}$. These catalogues are fundamental for the unique identification of objects. They are described by Paturel et al. (2003a).

Gas kinematics: this compilation of $\mathrm{H}$ I data associated with optical counterparts is the origin of the LEDA database (Paturel et al. 2003b). At present, the catalogue gathers 113086 measurements of a $\mathrm{HI}$ line width or a maximum rotation curve for 37377 galaxies. The catalogue is used to derive the homogenized physical maximum rotation velocity, VROT, corrected for inclination. Together with photometric information it is used to estimate distance using the Tully-Fisher relation (Tully \& Fisher 1977) for spiral galaxies.

Group membership: HyperLEDA also indexes multiple systems, including pairs, triplets, groups, and clusters. The catalogue collects information about groups and members of groups from the literature. The user interface allows us to determine the groups that an object is member of and, reciprocally, to find the members of a particular group. The database contains 19829 groups, and the compilation gives 391247 membership records for 370999 objects. In addition and independently of this, the web interface allows us to obtain a list of objects grouped to a specific target on the basis of proximity in projection on the sky and in redshift space, using the algorithm described by Prugniel et al. (1999).

$\mathrm{Mg}_{2}$ line strength indexes: the catalogue of published absorption-line $\mathrm{Mg}_{2}$ indices of galaxies and globular clusters (Golev \& Prugniel 1998) currently contains 9883 measurements for 3271 objects.

Morphology: the catalogue collects the RC3 numerical types, t, in de Vaucouleur's scale (de Vaucouleurs et al. 1991, see Sect. 3.3), which are combined with information on presence of bar, ring, multiplicity, or interaction to build the standard morphological classification. At the moment, HyperLEDA compiles 232305 morphological codes for 112572 objects. Most of these classifications result from a visual inspection of optical images. Recently an effort was made to calibrate an automatic classification algorithm and give a homogeneous classification for 4458 bright galaxies from the Sloan Digital Sky Survey (Baillard et al. 2011).

Nature: although HyperLEDA is intended to be a database of extragalactic objects, it is not possible to restrict it to galaxies alone. Often, the physical nature of an object is not known at the time of discovery, and it formally only appears as an extended source, while its extragalactic nature is merely a presumption. Other observations may confirm or change the earlier classification. Because the data are never excluded from the database, HyperLEDA contains virtually all kinds of celestial objects. The catalogue gives 5014711 nature classifications provided in the literature or made by the HyperLEDA team. The homogenization process combines the different classifications for each object, automatically chooses the most precise or assigns an undetermined nature in case of inconsistency. These cases are marked for human control to possibly solve the inconsistency. HyperLEDA identifies 3358587 galaxies and 372941 stars and objects of other nature.

Nuclear activity classification: this catalogue compiles information about the detection of signatures of activity in the centre of galaxies (active galactic nuclei, AGN, or star formation) for 88421 objects (Gavrilović et al. 2007). In

5 http://cdsweb.u-strasbg.fr/Dic/iau-spec.html 
particular, it contains the Véron catalogue of quasars and AGN $^{6}$ (Véron-Cetty \& Véron 2010).

Photometry: the catalogue presents the compilation of 28208556 apparent fluxes for 3605940 objects from the literature and from surveys. Mostly they are optical, near infrared, and Hi magnitudes. These values are stored as is, without correction for Galactic extinction or any other effects. This catalogue is supported with some aperture photometry (useful for calibrating observations of large nearby galaxies), photometric profiles, fitted growth curves, and colours (Prugniel \& Heraudeau 1998). The surface brightness is also given for 27761 galaxies. Paturel et al. (1994, 1997) described the reduction of apparent $B$ magnitude to the RC3-system, $B_{\mathrm{T}}$.

Redshift: the catalogue collects 2387020 redshifts in the heliocentric rest frame for 1296804 objects. The data are presented in units of $\mathrm{km} \mathrm{s}^{-1}$ as $c z$, where $z$ is the redshift and $c=299792.458 \mathrm{~km} \mathrm{~s}^{-1}$ is the speed of light. For low redshift, $z \ll 1$, the value $c z$ can be treated as the radial velocity of the object in the so-called optical convention. The homogenization of redshifts, transformation to different rest frames such as to the cosmic microwave background dipole or centroid of the Local Group as well as correction for Virgo infall is given by Paturel et al. (1997).

Sizes and position angles: these catalogues compile information on major and minor diameters of objects as well as the position angle of the major axis counted from north to east. The data are gathered for different pass-bands, various isophote levels, and different measurement techniques. The catalogue collects 11016356 diameter measurements for 3009469 objects. The position angle is known for 2763881 objects. The reduction to the standard system, corresponding to the size at the $25 \mathrm{~B}$-mag $\operatorname{arcsec}^{-2}$ isophote, is given by Paturel et al. (1991, 1997).

Spatially resolved kinematics of galaxies: the bibliographical catalogue assembles information on 15197 publications on rotation curve observations for 3860 galaxies (Prugniel et al. 1998).

Stellar kinematics: the catalogue, presented by Prugniel \& Simien (1996), contains 23797 observations of a central velocity dispersion for 16927 objects and 1668 measurements of maximum rotation of stellar populations for 832 earlytype galaxies. It is used in HyperLEDA to derive distances through the Faber-Jackson and the Fundamental Plane relations, after homogenization and combination with photometric data.

This article describes the catalogue of redshift-independent distance estimates.

The public mirrors of HyperLEDA are operated with the PLEINPOT software. Both the software and data are available as a free package ${ }^{7}$ for a Unix/Linux system, which enables installing a private mirror of the system. The web interface gives access to a number of tools. The principal ones are the searches by designation, near a position (also called cone searches), and by parameters using Structure Query Language (SQL)-like commands.

The search by designation prompts the user for the name of an object or a list of names. These names are searched for in the designation catalogue, taking into account a range of variants in their spelling (for example abbreviations or alternatives to the

\footnotetext{
6 http://heasarc.gsfc.nasa.gov/W3Browse/all/veroncat. html

7 http://leda.univ-lyon1.fr/install/mirror.html
}
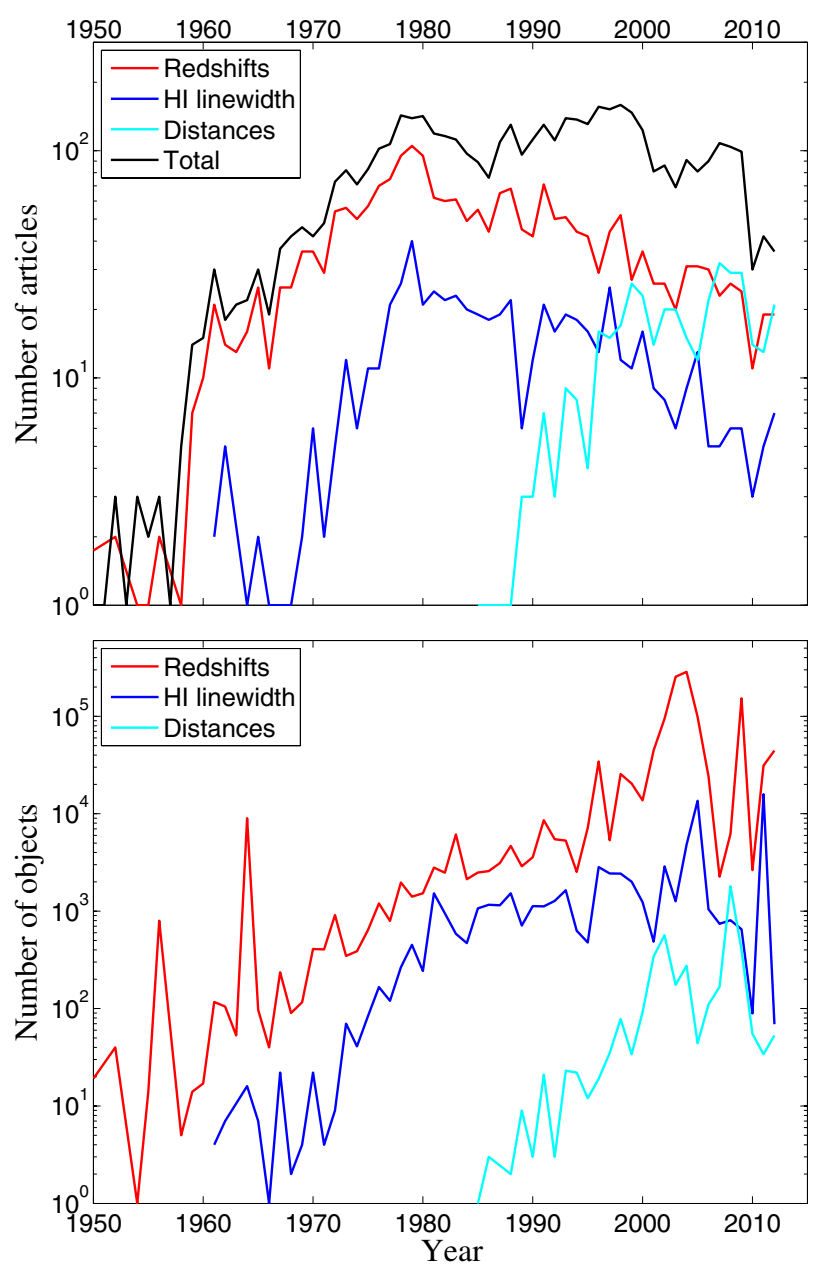

Fig. 1. Year-by-year statistics of data ingestion and processing in HyperLEDA. The top panel shows the number of processed articles per year. The black line corresponds to the whole database, while individual compilation catalogues are shown by different colours: the redshift catalogue is red, the HI line width is blue, and the distance catalogue is cyan. The bottom panel shows the number of individual objects processed in the given year.

catalogue acronym) and accepting some wildcard characters. In addition, a specific syntax allows searching for the nearest object to a given position on the sky. The search can be applied to the LEDA catalogue or to any of the other compilations.

The cone search takes a position on the sky and a radius to limit the surrounding area. A variety of images of the field can be displayed with a possible overlay that the database objects use, for instance, the Aladin ${ }^{8}$ applet (Bonnarel et al. 2000) from the Centre de Données de Strasbourg.

The SQL search gives a flexible access to the LEDA catalogue. The user can define her or his own SQL constraints and retrieve any desired fields or valid SQL expressions involving fields.

Some other search modes and tools are also available from the interface, but are not described in this short introduction.

Figure 1 illustrates the year by year statistics on data ingestion and processing in HyperLEDA. The top panel shows the number of surveyed articles sorted by on the year of publication for the whole database and for some compilations, namely the redshift, the gas kinematics, and the distance, which last is

8 http://aladin.u-strasbg.fr/ 

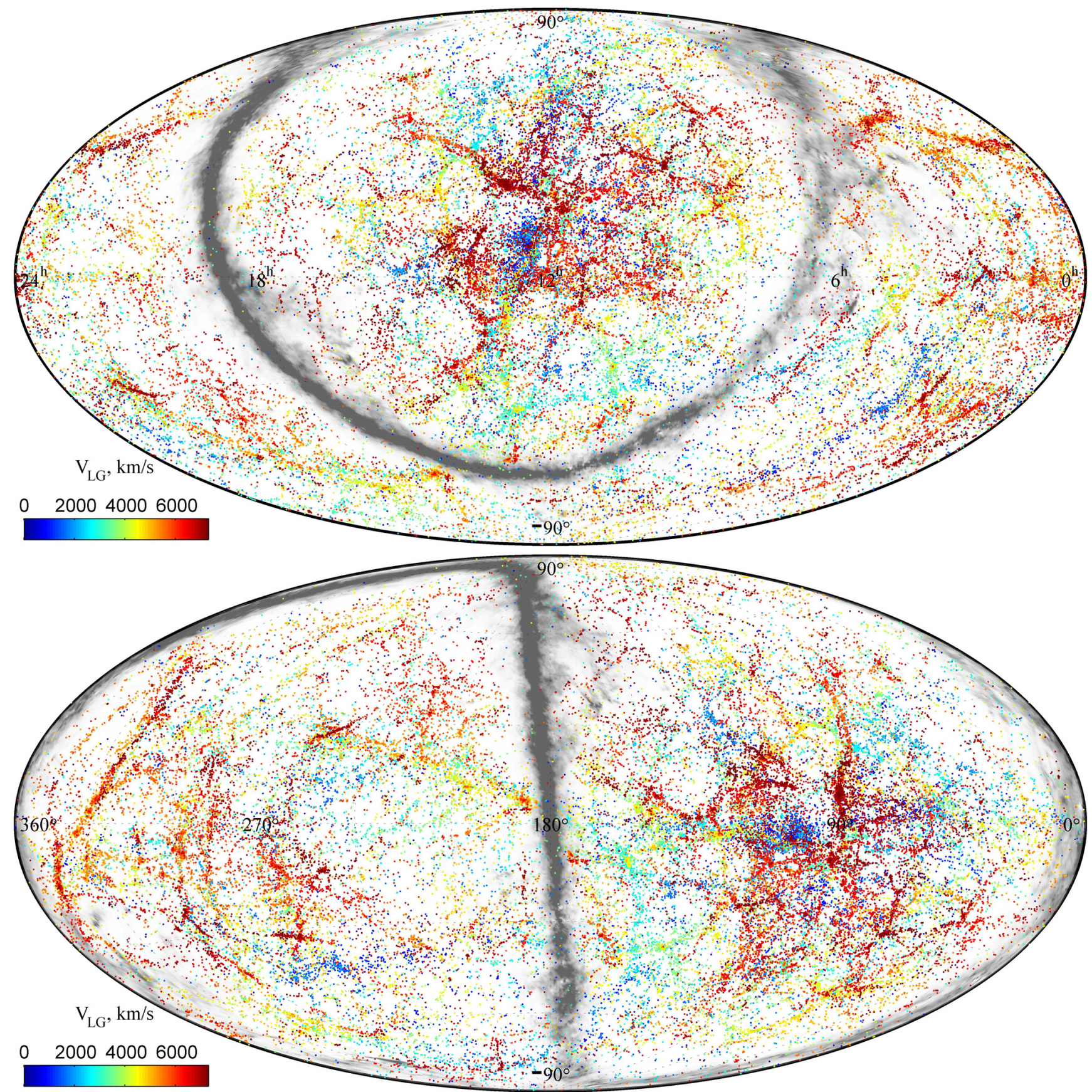

Fig. 2. All-sky distribution of about 60000 galaxies within $100 \mathrm{Mpc}$ from HyperLEDA in the equatorial (top panel) and the supergalactic coordinates (bottom panel). The galaxy redshifts are colour-coded from blue for nearby objects to brown for distant ones. The Zone of Avoidance in the Milky Way is shown by the grey clumpy clouds. The darkest regions correspond to the highest absorption, according to the Schlegel et al. (1998) extinction map.

the object of this article. The bottom panel shows the number of added objects in one year.

Figure 2 illustrates the distribution on the sky of the galaxies with known redshift up to $V_{\mathrm{LG}}<7300 \mathrm{~km} \mathrm{~s}^{-1}$, which corresponds to a distance of about $100 \mathrm{Mpc}$. $V_{\mathrm{LG}}$ denotes the radial velocity with respect to the Local Group centroid, as defined in the HyperLEDA online documentation. The colour of the symbols indicates the redshift. The map exhibits the wellknown filamentary structure of galaxy distribution, which connects the massive clusters and depicts the low-density regions. A detailed description of structures in a similar volume of the local Universe was recently carried out by Courtois et al. (2013). Despite the modern surveys, data in the Zone of Avoidance at low Galactic latitudes are highly incomplete. Galaxy extinction is shown as a grey clumpy belt in Fig. 2. The footprints of the different galaxy surveys are barely detectable, which confirms that in the nearby Universe the coverage of the sky by different redshift surveys is reasonably uniform (i.e. the completeness is about the same in any direction, except for the Galactic plane). In contrast, farther away than $100 \mathrm{Mpc}$, the sky coverage is not uniform because some regions are surveyed to a deeper level than others. In particular, the catalogue is much more complete in 
the region surveyed by the Sloan Digital Sky Survey (Ahn et al. 2013) than in the rest of the sky. Despite this, the general structures such as walls, filaments, clusters, and voids are still visible, but statistical uses of the database should take into account these various selection biases.

\section{Description of the distance catalogue}

\subsection{Organisation of the compilation}

The distance catalogue in HyperLEDA collects the redshiftindependent distance measurements published in the literature. It can be either original distance measurements, re-calibrations, or even compilations of data. Since it is difficult with limited resources to maintain an up-to-date complete literature survey, we are prioritizing the high-quality measurements that are potentially the most interesting standards for calibrating other indicators. The inclusion of lower priority datasets may be delayed by a few years.

An important step in adding a new source of data to the catalogue is to cross-identify its objects with HyperLEDA. An object in a publication is usually identified by one or several names and by coordinates (when the IAU recommendations are followed). In most cases it allows an automatic identification when the position and designations consistently match a unique object in HyperLEDA. In the opposite case, when there is no correspondence, a new object is added into the database. However, when the information is inconsistent (e.g. the position does not correspond to some of the designations), or when several HyperLEDA objects match the specification, manual handling is required. Although these cases are quite rare, typically $0.5-1 \%$ for the distance catalogue, the manual cross-identification consumes most of the human resource required for maintaining the database.

The goal of the cross-identification is to associate a unique internal identification number (PGC, see below) with each object in the new article. Then, to add the new data in HyperLEDA, we edit and execute ingestion rules that describe how to convert the published data into the internal HyperLEDA storage. This conversion involves very little alteration, for example, the transformation of a published linear distance to a stored distance modulus.

In addition to the cross-identification, the other most timeconsuming task is to code a description of the measurement protocol. This meta-information is essential for the subsequent measurement homogenization. This information is stored in a specific table where each entry describes a calibration used for distance measurements (the calibration parameter table, see below).

\subsection{Structure of the catalogue}

The distance catalogue currently consists of two main blocks: the measurement and the calibration tables.

The measurement table stores the actual published distance determinations, merging the data from the all sources in a single table. The fields of the table are chosen to keep the published values as completely as possible with minimal changes and to maintain an easy traceability back to the original paper.

The calibration table contains ancillary information describing the calibration reference (zero point) of a series of measurements.

In addition to these tables, we use the HyperLEDA bibliographical reference catalogue for publication linkage. It is shared by all the HyperLEDA catalogues. Each article in the bibliographic table as far as possible is associated with its standard code, commonly called BIBCODE. This allows one to establish a connection with other databases, in particular with the SAO/NASA Astrophysical Data System ${ }^{9}$ (ADS), where the full text of the original publication is available. The bibliographic table also contains some information about the history of the addition of the reference to HyperLEDA.

Finally, meta-data tables provide the description of the catalogue itself as well as the specification of each its field (unit, label, short textual description, reference to documentation, and so on). This auxiliary information is used for proper visualisation of the data.

The end user receives the information from the distance catalogue tables gathered together on-the-fly in a single view. This approach allows us, on the one hand, to avoid redundancy of the stored data and, therefore, to avoid the risk of inconsistency, and, on the other hand, to improve the readability of information by minimizing references to a different tables.

\subsubsection{Measurement table}

The measurement table contains the following fields:

PGC is the Principal Galaxies Catalogue number, which was invented by Paturel et al. (1989). We use the standard identification schema of the HyperLEDA database. Each object has a unique number, used to link the data from the different catalogues, and, in particular, to its various designations. When a "search by name" is performed, the system first resolves the provided name into a PGC number, which in turn is used to access the data.

MODULUS, E_MODULUS contains a published distance modulus and its one-sigma measurement error in mag. It is a so-called true distance modulus, $(m-M)_{0}$, which is corrected for both the extinction in the our Galaxy and the absorption in the host galaxy. The web interface also shows the distance in the linear scale in units of Mpc.

QUALITY describes the quality of the data. It is divided in the two parts. The first one is a data-related set of flags: "uncertain" (:), "preliminary" (p), "low-limit" (>), or “compilation" (c). They are based on information provided by authors. The "uncertain" flag reduces the weight of the measurement by a factor 2 during the homogenization procedure. Other flags lead to elimination of the measurement from averaging if other data are available. The "reject" (!) code describes our HyperLEDA knowledge about the reliability of the data and discards erroneous measurements from the homogenization procedure.

IREF is an internal code for the publication. It points toward the general bibliographic table of HyperLEDA, which associates it with the ADS/CDS BIBCODE, when available, as well as with a short description of the article, including the first author and the year of the publication. This allows us to search the whole database by author or by a given paper.

METHOD codes the distance determination method. The list of methods is given in Sect. 3.3

CALIB points out a detailed information about the calibration relation used for a measurement. This field allows us to group the data obtained with the same calibration.

In addition to these fields, which are exposed to the users of the database, some additional fields are kept for internal use.

9 http://cdsads.u-strasbg.fr/ 
ORIGNAME is the name of the object as cited in the original reference. This allows us to trace a given measurement back to the source table.

NOTE contains important remarks on specific measurement.

Finally, some fields exposed to the user are derived from an onthe-fly calculation or are computed during the homogenization.

DISTANCE is the linear distance, in Mpc, computed from MODULUS. It is given for convenience.

MODC is the homogenized distance modulus after the calibration correction is applied (see Sect. 4). The best distance determination for a galaxy can be derived from a weighted average of the individual MODC.

\subsubsection{Calibration table}

The field CALIB links the measurement to the calibration table with the following fields:

CALIB is a unique code used to identify a specific distance calibration.

METHOD is a distance determination method as in the measurement table.

IREF is an internal bibliographical code of the article where the calibration is published.

NOTE contains miscellaneous remarks useful for understanding a given calibration.

\subsection{Distance determination methods}

To date, a number of distance determination methods have been invented. They vary in the class of objects used (e.g. Cepheids) and in the physical background (e.g. period-luminosity relation). The corresponding information is coded in the METHOD field of the measurement table. Below we briefly describe of these methods. We divide the list into three parts: (i) direct distance determination methods; (ii) use of specific stellar objects as a standard rule or a standard candle; and (iii) techniques based on scaling laws for galaxies.

Direct methods determine distances straight from the measurement data and do not depend on external calibrations. They are the basis for constructing the cosmic distance ladder. The most important distance estimates use the trigonometric parallax of individual stars. The methods of statistical parallax and moving cluster parallax allow us to derive distances for groups of stars. It is very useful for calibrating methods based on the luminosity of the Cepheids and RR Lyrae. Unfortunately, these methods are usually restricted to our Galaxy or to its nearby satellites. A notable exception is NGC 4258, whose precise "maser" distance of $7.6 \mathrm{Mpc}$ (see hereafter) is precious for calibrating the other methods.

DEB: the detached eclipsing binaries (DEB) provide an accurate geometric method for distance determination. The fundamental parameters of the stars (the radii, effective temperatures, masses, and luminosities) can be determined from the light and radial velocity curves of an eclipsing binary. This method is independent of any intermediate calibration steps.

EPM, ESM: the expanding photosphere method (EPM) and the expanding shock-front method (ESM) are a geometric distance determination technique based on comparing radial velocities with proper motion of an expanding shell after a supernova explosion.
Maser: the method is based on studying kinematics of an accretion disk around a supermassive black hole by a radio maser emission. It gives a direct geometric estimate of an absolute distance. Humphreys et al. (2013) measured the distance of $7.6 \mathrm{Mpc}$ with $3 \%$ uncertainty to the Seyfert II galaxy NGC 4258 using ten years observations of the $\mathrm{H}_{2} \mathrm{O}$ maser.

A wide number of methods uses individual objects or stellar populations in the galaxies for distance determination. This class contains some of the most precise and important distance indicators for extragalactic astronomy: the Cepheids and RR Lyrae variable stars, the tip of the red giant branch (TRGB) and the horizontal branch (HB) stars. These distance indicators can be calibrated using the direct methods described above ${ }^{10}$. Except for SN Ia, all these methods are only effective for the nearby Universe on a scale from several to a few dozen Mpc.

BBSLF, BRSLF, BS, BS3B, BS3R: all these methods use the luminosity of the brightest stars in galaxies as standard candles. BBSLF and BRSLF consider the luminosity function of the brightest blue and red stars. BS3B and BS3R take the mean absolute magnitude of the three brightest blue or red stars. The luminosity of the brightest blue and red supergiants depends on the magnitude of the parent galaxy (Rozanski \& Rowan-Robinson 1994).

Cepheids: this is one of the most important standard candles. The method is based on the period-luminosity (PL) relation for Cepheid variable stars. There are many calibrations of the relation in different pass-bands using the Galactic or Large Magellanic Cloud (LMC) PL relation, for example, the Hubble Space Telescope key project on the extragalactic distance scale (Freedman et al. 2001), the HIPPARCOS trigonometric parallaxes (Feast \& Catchpole 1997), or the Baade-Wesselink methods (Storm et al. 2011). The calibration will be dramatically improved in the coming years thanks to the Gaia astrometric satellite, which starts to operate now.

CMD: this uses various features of the composite colourmagnitude diagram (CMD) of a galaxy resolved into individual stars to estimate the distance by comparison with template CMD or with theoretical isochrones. For instance, Dolphin (2000) developed the software that fits the observed CMD with synthetic data to simultaneously estimate the distance and the star formation history of a galaxy.

CS: the carbon-rich stars (CS) in the TP-AGB phase form the horizontal red tail on the CMD, and are about $0.5 \mathrm{mag}$ brighter than the TRGB. Battinelli \& Demers (2005) found the absolute $I$-band magnitude of $\mathrm{CS}$ as a function of the metallicity of the parent galaxy: $\left\langle M_{I}\right\rangle=-4.33+$ $0.28[\mathrm{Fe} / \mathrm{H}]$.

FGLR: the flux-weighted gravity-luminosity relationship (FGLR) is a technique to derive the distance from a spectral analysis of the B and A supergiant stars (Kudritzki et al. 2008). It is based on a tight correlation between the absolute bolometric magnitude and the flux-weighted gravity, $g / T_{\mathrm{eff}}^{4}$.

GCLF: the old globular cluster luminosity function (GCLF) method uses the peak (or turnover, TO) of the GCLF as a standard candle. For instance, Di Criscienzo et al. (2006) derived $M_{V, \mathrm{TO}}=-7.66 \pm 0.09$ with an adopted $\mathrm{LMC}$ distance modulus of 18.50 .

10 The references cited in the description of each method are examples of recent studies. A wider overview can be obtain in the body of the catalogue. 
GCR: the median of the globular cluster half-light radii (GCR) of $2.7 \pm 0.3$ pc (Jordán et al. 2005, for example) can be used as a standard ruler for the distance estimate. The half-light radius of individual GC needs to be corrected for colour, surface brightness, and host galaxy colour.

$\mathrm{HB}, \mathrm{BHB}$ : these methods use the horizontal branch $(\mathrm{HB})$ or the blue horizontal branch (BHB) stars as standard candles. Carretta et al. (2000) reported the relation between absolute magnitude and metallicity of $\mathrm{HB}: M_{V}(\mathrm{HB})=(0.13 \pm$ $0.09)([\mathrm{Fe} / \mathrm{H}]+1.5)+(0.54 \pm 0.07)$.

MS: this method fits the position of the main sequence below the turn-off with theoretical isochrones or with template CMD. It is related to the CMD distance determination method.

Miras: mira Ceti stars are long-period variable stars in the asymptotic giant branch phase. Ita \& Matsunaga (2011), among others, derived the period-magnitude relations for Mira-like variables in the LMC using bolometric, near- and mid-infrared magnitudes.

PNLF: this method uses the sharp exponential truncation of the planetary nebulae luminosity function (PNLF) as a standard candle. The zero-point, $M^{*}=-4.48$, is based on the M 31 distance of $710 \mathrm{kpc}$ (Ciardullo et al. 2002).

$\mathrm{RC}$ : the red clump (RC) is populated by core helium-burning stars of intermediate age. Their mean absolute magnitude provides a standard candle for distance determination. Girardi \& Salaris (2001) found important non-linear dependences on both the age and the metallicity of the stellar population.

RRLyrae: the method is based on the mean absolute magnitude for RR Lyrae variable stars, which depends on metallicity: $M_{V}(\mathrm{HB})=(0.18 \pm 0.09)([\mathrm{Fe} / \mathrm{H}]+1.5)+(0.57 \pm 0.07)$ (Carretta et al. 2000).

RSV: this method uses the period-luminosity relation for the red supergiant variable (RSV) stars. The calibration of the PL relation by Pierce et al. (2000) adopts the distance modulus of 18.50 mag for LMC. The RSVs as well as the Miras are longperiod variable stars.

SBF: the surface brightness fluctuations (SBF) method relies on the luminosity fluctuations that arise from the counting statistics of stars that contribute to the flux in each pixel of an image (Tonry \& Schneider 1988). The absolute fluctuation magnitude depends on the stellar populations and, consequently, on the colour of the galaxy. It can only be applied to old stellar populations.

SNIa: because of their extremely high luminosity and regular behaviour, the type Ia supernovae (SN Ia) provide a powerful tool for measuring cosmological distances. The method uses the relationship between the light-curve shape and the maximum luminosity of a SN Ia.

TRGB: the tip of the red giant branch (TRGB) is an excellent distance indicator for nearby galaxies that are resolved into individual stars. The method, relying on the old stellar population, can be used for galaxies of any morphological types. Thanks to the shallow colour-dependence of the magnitude of the TRGB in the $I$ band, the method is one of the most precise distance indicators. For example, Rizzi et al. (2007) calibrated the zero-point of the TRGB method using HB stars: $M_{I}^{\mathrm{JC}}=-4.05( \pm 0.02)+0.22( \pm 0.01)[(V-I)-1.6]$.

Methods that are based on scaling relations are empirical relationships between the intrinsic luminosity of a galaxy and its properties such as kinematics, and surface brightness. The most important ones are the Tully-Fisher (TF) relation for spirals and the fundamental plane (FP) for early-type galaxies. Because the methods use the total luminosity of a galaxy as a standard candle, they can be applied on scales of up to several hundred Mpc. These methods provide only a low precision for individual measurements, but they give good results in a statistical sense with huge sets of data. This is especially true for the Tully-Fisher relation, where obtaining observational data is relatively inexpensive. The TF and FP methods allow us to investigate the cosmic flows in the Universe on scales of several hundred Mpc (Tully et al. 2013).

FJ: the Faber-Jackson (FJ) relation provides a standard candle for elliptical and early-type galaxies based on the relationship between absolute magnitude and central velocity dispersion.

FP: the fundamental plane (FP) is a distance determination method for early-type galaxies based on relation between the absolute magnitude, effective radius, velocity dispersion, and mean surface brightness. $\log D=\log r_{\mathrm{e}}-1.24 \log \sigma+$ $0.82 \log \langle I\rangle_{\mathrm{e}}+0.173$ (Kelson et al. 2000).

SB-M, Sersic-M: the methods using the surface brightness-total magnitude relation (SB-M) or the Sersic index-total magnitude relation (Sersic-M) can be considered as a rough distance estimate for small-mass elliptical galaxies.

Sosie: the method of "look-alike" (sosie in French) was proposed by Paturel (1984). It is based on the idea that galaxies with the same morphological type, the same inclination, and the same Hi line width must have the same absolute luminosity according to the TF relation.

TF, BTF: the Tully-Fisher (TF) method is a standard candle based on empirical relationship between the absolute magnitude of a spiral galaxy and its maximum rotational velocity, estimated by a H I line width. The recent calibration of $I$-band TF relation gives $M_{I}^{b, i, k}=-21.39-8.81\left(\log W_{\mathrm{mx}}^{i}-\right.$ 2.5) (Tully \& Courtois 2012).

The baryonic Tully-Fisher (BTF) relation uses the relationship between the amplitude of rotation and the baryonic mass of the galaxy. This relation takes into account not only the stellar light from optical data as in the original TF relation, but also the mass of gas in neutral and molecular forms. The BTF relation is similar to the TF for giant spiral galaxies, and it represents an improvement for dwarf galaxies with circular velocities below $90 \mathrm{~km} \mathrm{~s}^{-1}$ (McGaugh et al. 2000), where cold gas represents an important and variable dynamical component. The BTF can also be applied to gas-rich dwarf elliptical galaxies (De Rijcke et al. 2007).

\subsection{Content of the catalogue}

The distance catalogue currently contains 6640 distance measurements for 2335 galaxies compiled from 430 articles. We pay special attention to the Local Volume, $D \lesssim 10 \mathrm{Mpc}$, because the vast majority of high-precision distances are measured for nearby galaxies. For instance, at the moment, we gather 2594 distance moduli for 492 objects in this local part of the Universe, in particular, 1338 estimates for 347 galaxies based on the TRGB method. As it is illustrated in Fig. 3, most of the articles list measurements for a single or a few galaxies. The distance catalogue describes the specifics of the work on determining the distances to galaxies. Single galaxies are presented in 198 articles (50\%) and only 13 articles (3\%) give measurements for more than 50 galaxies.

The distribution on the sky of the galaxies with known redshift-independent distances is shown in Fig. 4. The clumpy 


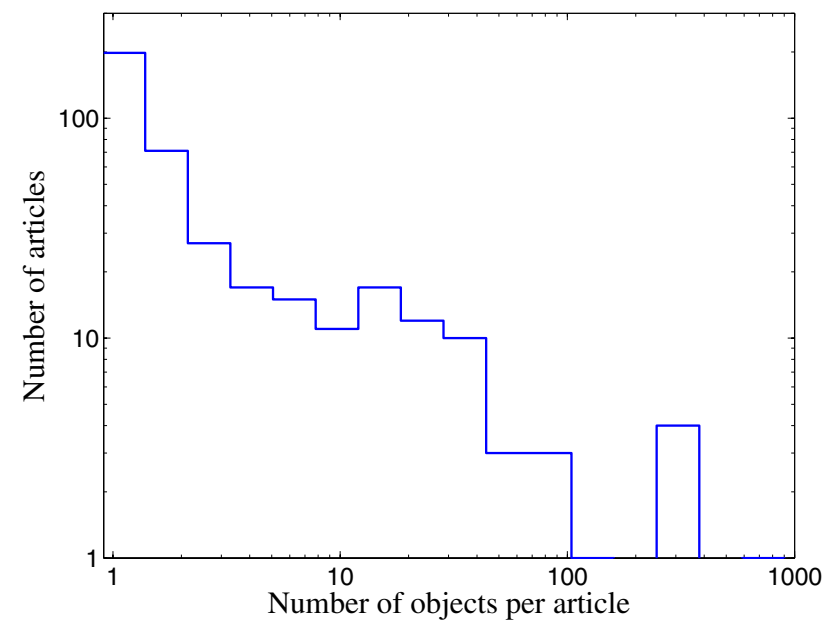

Fig. 3. Distribution of articles by the number of published galaxies for the distance catalogue.

grey belt represents the regions of strong extinction in our Galaxy (the Zone of Avoidance). The colour of the dots codes the distance to a galaxy, from blue for nearby objects to brown for the distant ones. Galaxies farther away than $20 \mathrm{Mpc}$ are shown as brown filled circles. The small black dots represent all galaxies with $V_{\mathrm{LG}}<3500 \mathrm{~km} \mathrm{~s}^{-1}$ (i.e. within $50 \mathrm{Mpc}$ ), roughly encompassing the Local Supercluster, whose core, the Virgo cluster, lies near the centre of the top-panel map (about $\mathrm{RA}=12 \mathrm{~h} \cdot 5$, Dec $=12^{\circ}$ ). Most of the galaxies are concentrated near the supergalactic plane, revealing the flat shape of the Local Supercluster, which is clearly seen in the supergalactic coordinates in the bottom panel of Fig. 4. Fortunately, the Virgo cluster is located near the Galactic north pole, and the sheet of the Local Supercluster galaxies is perpendicular to the plane of the Milky Way. Because the Local Group stands on the edge of the Local Supercluster, we do not see its extension in the direction opposite to the Virgo cluster.

Figure 5 illustrates the distribution of individual measurements by different methods as a function of the distance. The various shades of grey in the top panel distinguish the contribution of the most significant methods, namely the horizontal branch, the RR Lyrae, the tip of the red giant branch, the Cepheids, the surface brightness fluctuations, and the brightest stars. The first peak below $1 \mathrm{Mpc}$ corresponds to the Local Group galaxies. The second spike around 4-5 Mpc marks the systematic study of the Local Volume (Karachentsev et al. 2013), where the most prominent groups of galaxies lie around Cen A, M 81, IC 342 and several other giant galaxies are situated about 3-4 Mpc away from the Milky Way. McCall (2014) named this remarkable distribution "the Council of Giants". Beyond $5 \mathrm{Mpc}$, the sharp cut-off in the number of measurements reflects the increasing observational difficulty and cost of TRGB determinations. To reach the necessary limiting magnitude, several HST orbits are required for a single field. The third maximum, around $16-17 \mathrm{Mpc}$, has a physical origin. It corresponds to the centre of the Virgo cluster. The figure shows that the prevalence of the different indicators changes with the distance. HB and RR Lyrae stars can be used only inside the Local Group, below $1 \mathrm{Mpc}$. The tip of RGB is much brighter, $M_{I}=-4$, and the method is commonly used up to $10 \mathrm{Mpc}$, with extension up to about $20 \mathrm{Mpc}$ in particular cases. The use of Cepheids is much more expensive in terms of observations, because it requires time series. This explains the relatively small number of measurements, although it is one of the most precise and refined methods. Currently, the Cepheids allow us to confidently derive the distances up to $20 \mathrm{Mpc}$. The SBF method is not as precise as the previous ones, but an accuracy about $0.2 \mathrm{mag}$ in the distance modulus can be achieved for a wide range of distances up to $40-50 \mathrm{Mpc}$. The brightest-stars method cannot be considered as precise, but in many cases, it is the only possibility to estimate the distance between 5 to $10 \mathrm{Mpc}$. Farther out, the method based on the TF relation is the most effective. Recent calibrations give a scatter of about $0.34-0.38$ in the different band-passes (Tully \& Pierce 2000). HyperLEDA contains H I line widths for about 37000 galaxies. Taking into account the existence of photometry and limiting the inclination angle more than $45^{\circ}$, this allows us to estimate a TF distance for about 19000 galaxies on a scale of several hundred Mpc. The distribution of the galaxies with available data for TF distance estimate is shown in the bottom panel of Fig. 5 by grey diamonds for comparison. A similar distribution of galaxies with known redshift is displayed by open circles. This means that for large scales the TF distance estimate is possible only for a third of the known galaxies. In addition, black squares show the objects with precise distance measurements. It is clear that our knowledge on accurate distances is more or less complete only in the Local Volume on a scale of up to 5-7 Mpc.

\section{Distance homogenization}

The measurements collected in this catalogue form an inhomogeneous data set where each individual publication uses a specific distance scale and is affected by its own systematics. As for the other characteristics of galaxies, HyperLEDA aims to compensate these discrepancies. The homogenization in HyperLEDA attempts to reduce these effects as much as possible.

The strategy adopted in the present version of the catalogue is the following: First, we define a set of calibrators that determines our distance scale. Then, we apply zero-point correction accounts for the shift between a given calibration system and our adopted distance scale.

These different steps are described below.

\subsection{Distance standards}

The goal is to define a set of galaxies with precise distance measurements that can be used to calibrate other systems. This will define our own distance scale.

The most recent and widely adopted distance scale is based on Cepheid measurements in 31 galaxies reported in the HST key project (Freedman et al. 2001). The quality of the data are still not surpassed, but the small size of the sample limits its usage for calibrating other methods and datasets. To circumvent this limitation, we can consider building our calibration set around the "CMDs/TRGB" catalogue (Jacobs et al. 2009) of EDD, encompassing highly homogenized measurements for 305 galaxies using the maximum-likelihood TRGB fit by Makarov et al. (2006) and the TRGB zero-point by Rizzi et al. (2007).

Table 1 presents a comparison of different data samples with the EDD reference sample. Column 1 gives the reference of the datasets. Column 2 is the number of common objects compared with our EDD sample. Columns 3 and 4 present the mean bias and its one-sigma uncertainty. Column 5 shows the dispersion around the bias. Column 6 gives an additional description of the compared measurements. The datasets are grouped by different distance determination methods. 

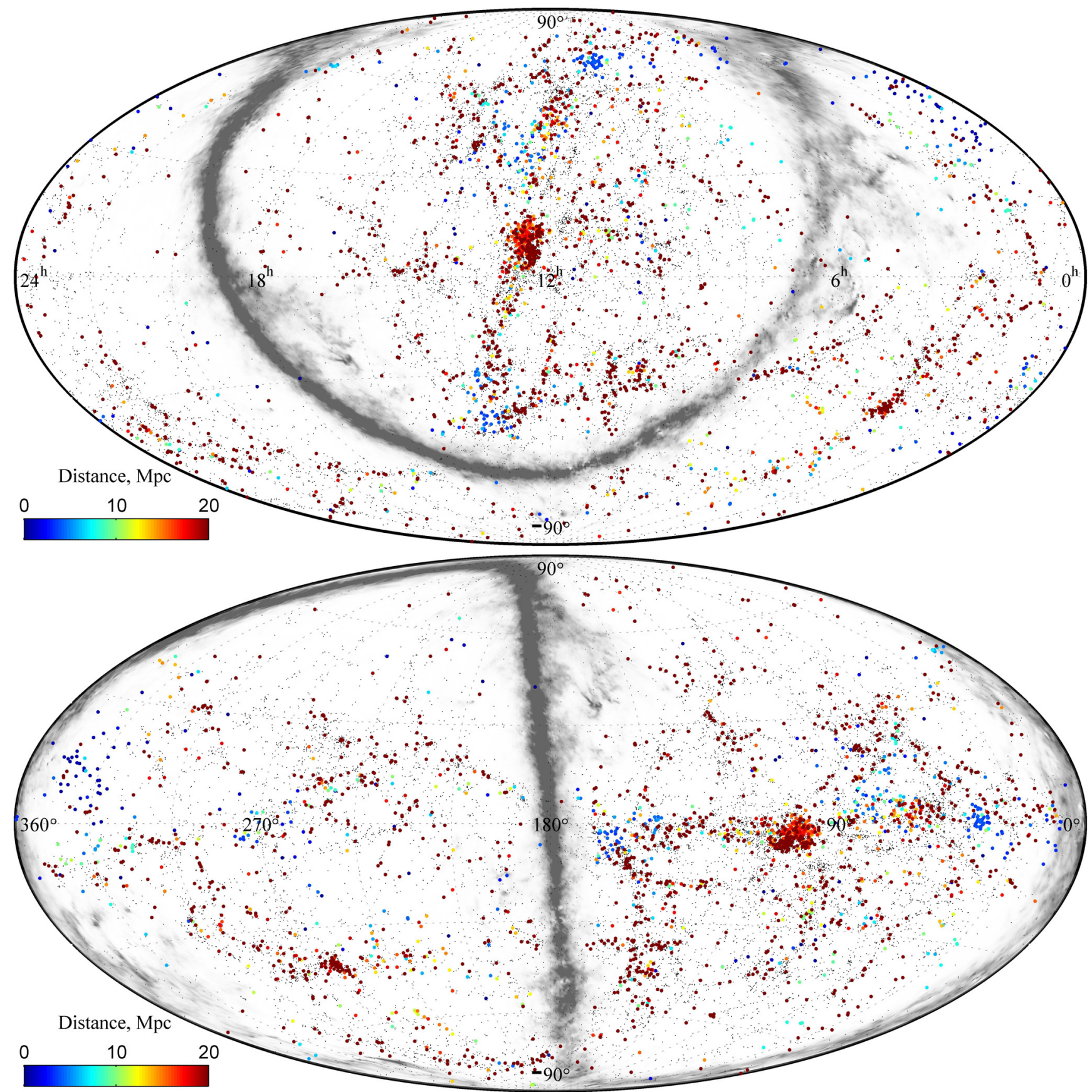

Fig. 4. All-sky distribution of galaxies with known redshift-independent distance estimate in equatorial (top panel) and in supergalactic coordinates (bottom panel). The distance is shown by colour from nearby (blue) to distant (brown). The small black dots represent the distribution of galaxies on a scale of $40 \mathrm{Mpc}$. The zone of high extinction in the Milky Way is indicated in grey.

The table gives a first taste of the intrinsic precision of individual methods. The TRGB and Cepheids distances have a precision of about $0.1 \mathrm{mag}$. The SBF and PNLF methods are precise to $0.2 \mathrm{mag}$. Finally, brightest stars are only precise to $0.6 \mathrm{mag}$. Therefore, this method is useful only when no other measurement available.

While the TRGB method displays a very low internal dispersion (0.06 mag), the various measurement sets are not fully independent because they are often based on the same original images and differ only by some aspects of the analysis and calibration. Low dispersion indicates that the photometric errors probably outweigh the systematics associated with the different techniques of tip detection.

The comparisons gathered in Table 1 reveal a fair consistency, generally within two sigma. Nevertheless, the small -0.02 mag bias between any of the considered TRGB sets and the "CMDs/TRGB" sample appears to be statistically significant. Its origin is not clear. A comparison of the TRGB method with various period-luminosity relations for Cepheids shows that they agree excellently. In most cases, the average difference does not exceed $0.02 \mathrm{mag}$. In other words, the TRGB and Cepheids distance scales are consistent on a level better than $1 \%$. Note 
Table 1. Comparison of different sets of distance determinations with the TRGB set from EDD.

\begin{tabular}{|c|c|c|c|c|}
\hline Sample & $N$ & $\left\langle\mu-\mu_{\mathrm{EDD}}\right\rangle$ & $\sigma$ & Notes \\
\hline \multicolumn{5}{|l|}{ TRGB } \\
\hline Rizzi et al. (2007) excluding EDD & 83 & $-0.016 \pm 0.008$ & 0.060 & Rizzi et al. (2007) \\
\hline Makarov et al. (2013) & 26 & $-0.022 \pm 0.005$ & 0.025 & Rizzi et al. (2007) \\
\hline Radburn-Smith et al. (2011) & 13 & $-0.021 \pm 0.024$ & 0.086 & Rizzi et al. (2007) \\
\hline Dalcanton et al. (2009) & 87 & $-0.064 \pm 0.008$ & 0.062 & Padova isochrones \\
\hline$M_{I}(\mathrm{TRGB})=-4.05$ & 181 & $-0.027 \pm 0.006$ & 0.062 & \\
\hline Lee et al. (1993) & 30 & $-0.073 \pm 0.021$ & 0.114 & \\
\hline \multicolumn{5}{|l|}{ Cepheids } \\
\hline Saha et al. (2006) & 15 & $-0.013 \pm 0.048$ & 0.157 & \\
\hline Fouqué et al. (2003) & 7 & $+0.002 \pm 0.041$ & 0.103 & \\
\hline Kanbur et al. (2003) & 8 & $+0.003 \pm 0.062$ & 0.197 & \\
\hline Tammann et al. (2003) & 7 & $+0.063 \pm 0.047$ & 0.110 & \\
\hline Freedman et al. (2001) & 13 & $-0.065 \pm 0.030$ & 0.100 & \\
\hline Freedman et al. (2001) & 14 & $-0.016 \pm 0.034$ & 0.143 & metallicity corrected \\
\hline Lanoix et al. (1999) & 16 & $-0.029 \pm 0.044$ & 0.147 & \\
\hline Udalski et al. (1999) & 8 & $+0.015 \pm 0.061$ & 0.196 & corrected to $\mu_{\mathrm{LMC}}=18.50$ \\
\hline Gieren et al. (1998) & 7 & $-0.005 \pm 0.047$ & 0.109 & \\
\hline Madore \& Freedman (1991) & 13 & $+0.007 \pm 0.035$ & 0.117 & \\
\hline \multicolumn{5}{|l|}{ 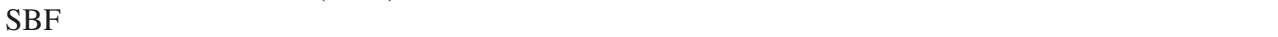 } \\
\hline Jensen et al. (2003) & 8 & $-0.223 \pm 0.076$ & 0.223 & \\
\hline Tonry et al. (2000) & 16 & $+0.092 \pm 0.057$ & 0.250 & \\
\hline \multicolumn{5}{|l|}{ PNLF } \\
\hline Ciardullo et al. (1989) & 10 & $+0.043 \pm 0.089$ & 0.225 & \\
\hline \multicolumn{5}{|l|}{ Brightest stars } \\
\hline Brightest blue stars & 61 & $-0.039 \pm 0.076$ & 0.586 & \\
\hline Brightest red stars & 21 & $-0.080 \pm 0.140$ & 0.632 & \\
\hline
\end{tabular}

that the TRGB calibration by Rizzi et al. (2007) is based on the luminosity of the horizontal branch reported by Carretta et al. (2000) and is independent of Cepheids distances. Although the SBF method is calibrated on the Cepheid variables it requires a large correction to be consistent with other methods.

In summary, the CMDs/TRGB catalogue (Jacobs et al. 2009) can be considered as the basis for the standard sample of galaxies for the distance homogenization. Many authors point out that the TRGB method has a precision similar to that of the Cepheids (Sakai et al. 1996; Bellazzini et al. 2001). Rizzi et al. (2007) found an excellent agreement between TRGB and Cepheid scales $\left(\mu_{\mathrm{Ceph}}-\mu_{\mathrm{TRGB}}=-0.01 \pm 0.03\right)$ using 15 nearby galaxies. Our analysis confirms this conclusion. We selected 194 galaxies with an uncertainty better than 0.1 mag from the CMDs/TRGB catalogue to be the core of our calibration set. This sample covers nearby galaxies well, but is still limited for calibrations of long-distance methods. As can be seen from Fig. 5, current TRGB measurements are mostly bounded by a distance of 5-6Mpc. This restriction is especially strong for early-type galaxies because the Local Volume is almost free of giant ellipticals. To partially palliate this problem we adopted 29 measurements with an uncertainty better than 0.12 mag reported Freedman et al. (2001, corrected for metallicity effect) from the final results of the Hubble Space Telescope key project. It is interesting to note that excluding two galaxies, NGC 5253 and IC 1613 with measurement errors of 0.14 and 0.15 mag, makes the accordance between TRGB and Cepheids scales ideal, $\left\langle\mu_{\mathrm{KP}}-\mu_{\mathrm{EDD}}\right\rangle_{w}=-0.001 \pm 0.034$. This dataset effectively extends to $15-20 \mathrm{Mpc}$, favouring spiral galaxies. We include a new geometric maser distance, $D=7.6 \mathrm{Mpc}$ or $\mu=29.40 \mathrm{mag}$, to NGC 4258 (Humphreys et al. 2013), which agree excellently with the TRGB estimate $\mu_{\mathrm{TRGB}}=29.42 \pm 0.04$ (Jacobs et al. 2009) and with the Freedman et al. (2001) value of $\mu_{\text {Ceph }}=$ $29.44 \pm 0.07$ mag. Our sample of the standard distance measurements contains 211 galaxies.

\subsection{Calibration correction}

Most of the data in the catalogue refer to the calibration used to determine the distance. It allows us to group measurements made with the same method and the same calibration. For each calibration, we determine the mean bias as the weighted average of the difference between individual measurements and our standard distance set. We apply this correction to data if an intersection with the standard distance set has at least five objects. Now, the bias is estimated for 58 calibrations of 13 distance determination methods. Field MODC shows the homogenized distance modulus for individual measurements after the calibration correction is applied.

\section{Conclusion}

Over the past decade, the number of available data in extragalactic astronomy has increased enormously, thanks to modern surveys. However, despite the increase in quality of the measurement, when a sample for calibrating of distance indicators needs to be established, it remains necessary to critically review a large number of various sources of measurements. The systematics that affect specific methods or specific series of measurements have to be clearly understood, and the need for well-documented compilations remains as acute as ever. Fortunately, the access and the tools to handle the published data are improving, which keeps the effort at a sustainable level.

We here presented the compilation catalogue of redshiftindependent distances in the HyperLEDA database. Currently, we have surveyed 430 publications that yield 6640 distance estimates for 2335 galaxies. Most of them are concentrated in the Local Volume. Each individual data series is recalibrated to a common distance scale based on a carefully selected set of high-quality measurements. In addition, the large collections of 

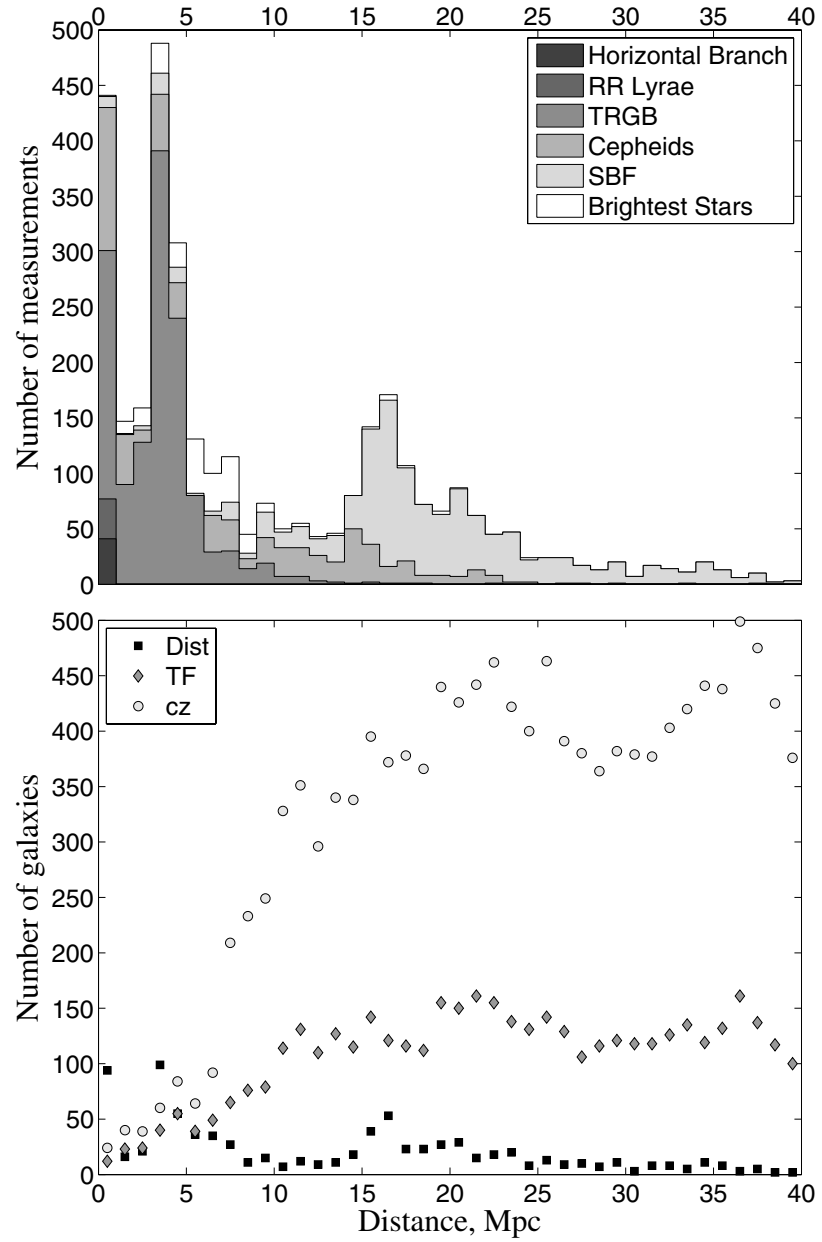

Fig. 5. Top panel: distribution of the individual distance estimates versus distance. Only the five most significant methods, namely the horizontal branch, the RR Lyrae, the tip of the red giant branch (TRGB), the Cepheids, the surface brightness fluctuations (SBF), and the brightest stars, were considered to build the histogram. While the outer envelope shows the total number of measurements in each $1 \mathrm{Mpc}$ bin, the different grey tones distinguish the contribution of each method. Bottom panel: distribution of the individual galaxies. The black squares correspond to objects with known redshift-independent distance estimate (Dist), excluding the scaling relations. The distributions of galaxies with TullyFisher data (TF) is shown by grey diamonds, and galaxies with known redshift, $c z$, are illustrated by open circles. TF is computed using the $\mathrm{HI}$ and photometric parameters of HyperLEDA, while the redshift is transformed into distance using the Hubble law.

H I and photometric data in HyperLEDA enable distance estimates for 19000 spirals using the TF relation.

The distance catalogue is available through the web-interface of the HyperLEDA database ${ }^{11}$, which is currently maintained by the Observatoire de Lyon (France) and the Special Astrophysical Observatory of the Russian Academy of Sciences (Russia).

Acknowledgements. We are thankful to Georges Paturel for a long-standing support and interest in the project, and to Chantal Petit for years of work on HyperLEDA. We appreciate the help and contribution in software development of Rumen Bogdanovski, Sergey Koposov, Vladimir Georgiev, Igor Chilingarian, and Ivan Zolotukhin. We are grateful for catalogue support to Anatoly Zasov, Valeri Golev, and Natasa Gavrilovic. We thank Mina Koleva and Galina Korotkova for data mining, analysis, and processing. We thank the numerous users who report missing or erroneous data, make suggestions to improve the service, and provide encouragement. D.M. acknowledges the support of the

\footnotetext{
${ }^{11}$ http://leda.univ-lyon $1 . f r$
}

Russian Science Foundation grant 14-12-00965. D.M. thanks Observatoire de Lyon for hospitality during the work.

\section{References}

Ahn, C. P., Alexandroff, R., Allende Prieto, C., et al. 2014, ApJS, 211, 17 Baillard, A., Bertin, E., de Lapparent, V., et al. 2011, A\&A, 532, A74 Battinelli, P., \& Demers, S. 2005, A\&A, 442, 159

Bellazzini, M., Ferraro, F. R., \& Pancino, E. 2001, ApJ, 556, 635

Bonnarel, F., Fernique, P., Bienaymé, O., et al. 2000, A\&AS, 143, 33

Carretta, E., Gratton, R. G., Clementini, G., \& Fusi Pecci, F. 2000, ApJ, 533, 215

Ciardullo, R., Jacoby, G. H., Ford, H. C., \& Neill, J. D. 1989, ApJ, 339, 53

Ciardullo, R., Feldmeier, J. J., Jacoby, G. H., et al. 2002, ApJ, 577, 31

Courtois, H. M., Tully, R. B., Fisher, J. R., et al. 2009, AJ, 138, 1938

Courtois, H. M., Tully, R. B., \& Héraudeau, P. 2011, MNRAS, 415, 1935

Courtois, H. M., Hoffman, Y., Tully, R. B., \& Gottlöber, S. 2012, ApJ, 744, 43

Courtois, H. M., Pomarède, D., Tully, R. B., Hoffman, Y., \& Courtois, D. 2013, AJ, 146, 69

Dalcanton, J. J., Williams, B. F., Seth, A. C., et al. 2009, ApJS, 183, 67

De Rijcke, S., Zeilinger, W. W., Hau, G. K. T., Prugniel, P., \& Dejonghe, H. 2007, ApJ, 659, 1172

de Vaucouleurs, G., de Vaucouleurs, A., Corwin, Jr., H. G., et al. 1991, 3d Reference Catalogue of Bright Galaxies. Volume I: Explanations and references. Volume II: Data for galaxies between $0^{\mathrm{h}}$ and $12^{\mathrm{h}}$. Volume III: Data for galaxies between $12^{\mathrm{h}}$ and $24^{\mathrm{h}}$.

Di Criscienzo, M., Caputo, F., Marconi, M., \& Musella, I. 2006, MNRAS, 365, 1357

Dolphin, A. E. 2000, ApJ, 531, 804

Feast, M. W., \& Catchpole, R. M. 1997, MNRAS, 286, L1

Fouqué, P., Storm, J., \& Gieren, W. 2003, in Stellar Candles for the Extragalactic Distance Scale, eds. D. Alloin \& W. Gieren, Lect. Notes Phys. (Berlin: Springer Verlag), 635, 21

Freedman, W. L., Madore, B. F., Gibson, B. K., et al. 2001, ApJ, 553, 47

Gavrilović, N., Mickaelian, A., Petit, C., Popović, L. Č., \& Prugniel, P. 2007, in Black Holes from Stars to Galaxies - Across the Ranges of Masses, eds. V. Karas, \& G. Matt, IAU Symp., 238, 371

Gieren, W. P., Fouqué, P., \& Gómez, M. 1998, ApJ, 496, 17

Girardi, L., \& Salaris, M. 2001, MNRAS, 323, 109

Golev, V., \& Prugniel, P. 1998, A\&AS, 132, 255

Hipparchus, N. about 150 BC, Peri megethon kai apostematon

Hubble, E. P. 1926, ApJ, 64, 321

Hubble, E. 1929, Proc. National Academy of Science, 15, 168

Humphreys, E. M. L., Reid, M. J., Moran, J. M., Greenhill, L. J., \& Argon, A. L. 2013, ApJ, 775, 13

Ita, Y., \& Matsunaga, N. 2011, MNRAS, 412, 2345

Jacobs, B. A., Rizzi, L., Tully, R. B., et al. 2009, AJ, 138, 332

Jarosik, N., Bennett, C. L., Dunkley, J., et al. 2011, ApJS, 192, 14

Jensen, J. B., Tonry, J. L., Barris, B. J., et al. 2003, ApJ, 583, 712

Jordán, A., Côté, P., Blakeslee, J. P., et al. 2005, ApJ, 634, 1002

Kanbur, S. M., Ngeow, C., Nikolaev, S., Tanvir, N. R., \& Hendry, M. A. 2003, A\&A, 411, 361

Karachentsev, I. D., Karachentseva, V. E., Huchtmeier, W. K., \& Makarov, D. I. 2004, AJ, 127, 2031

Karachentsev, I. D., Kashibadze, O. G., Makarov, D. I., \& Tully, R. B. 2009, MNRAS, 393, 1265

Karachentsev, I. D., Makarov, D. I., \& Kaisina, E. I. 2013, AJ, 145, 101

Kelson, D. D., Illingworth, G. D., Tonry, J. L., et al. 2000, ApJ, 529, 768

Kudritzki, R.-P., Urbaneja, M. A., Bresolin, F., et al. 2008, ApJ, 681, 269

Lanoix, P., Paturel, G., \& Garnier, R. 1999, MNRAS, 308, 969

Lee, M. G., Freedman, W. L., \& Madore, B. F. 1993, ApJ, 417, 553

Madore, B. F., \& Freedman, W. L. 1991, PASP, 103, 933

Makarov, D., Makarova, L., Rizzi, L., et al. 2006, AJ, 132, 2729

Makarov, D. I., Makarova, L. N., \& Uklein, R. I. 2013, Astrophys. Bull., 68, 125

McCall, M. L. 2014, MNRAS, 440, 405

McGaugh, S. S., Schombert, J. M., Bothun, G. D., \& de Blok, W. J. G. 2000, ApJ, 533, L99

Paturel, G. 1984, ApJ, 282, 382

Paturel, G., Bottinelli, L., Fouque, P., \& Gouguenheim, L. 1988, in European Southern Observatory Conference and Workshop Proc., eds. F. Murtagh, A. Heck, \& P. Benvenuti, 28, 435

Paturel, G., Fouque, P., Bottinelli, L., \& Gouguenheim, L. 1989, A\&AS, 80, 299

Paturel, G., Garcia, A. M., Fouque, P., \& Buta, R. 1991, A\&A, 243, 319

Paturel, G., Bottinelli, L., \& Gouguenheim, L. 1994, A\&A, 286, 768

Paturel, G., Andernach, H., Bottinelli, L., et al. 1997, A\&AS, 124, 109

Paturel, G., Petit, C., Prugniel, P., et al. 2003a, A\&A, 412, 45

Paturel, G., Theureau, G., Bottinelli, L., et al. 2003b, A\&A, 412, 57

Perlmutter, S., Aldering, G., Goldhaber, G., et al. 1999, ApJ, 517, 565 
A\&A 570, A13 (2014)

Pierce, M. J., Jurcevic, J. S., \& Crabtree, D. 2000, MNRAS, 313, 271

Prugniel, P., \& Heraudeau, P. 1998, A\&AS, 128, 299

Prugniel, P., \& Simien, F. 1996, A\&A, 309, 749

Prugniel, P., Zasov, A., Busarello, G., \& Simien, F. 1998, A\&AS, 127, 117

Prugniel, P., Golev, V., \& Maubon, G. 1999, A\&A, 346, L25

Radburn-Smith, D. J., de Jong, R. S., Seth, A. C., et al. 2011, ApJS, 195, 18

Riess, A. G., Filippenko, A. V., Challis, P., et al. 1998, AJ, 116, 1009

Rizzi, L., Tully, R. B., Makarov, D., et al. 2007, ApJ, 661, 815

Rozanski, R., \& Rowan-Robinson, M. 1994, MNRAS, 271, 530

Saha, A., Thim, F., Tammann, G. A., Reindl, B., \& Sandage, A. 2006, ApJS, 165,108

Sakai, S., Madore, B. F., \& Freedman, W. L. 1996, ApJ, 461, 713

Schlegel, D. J., Finkbeiner, D. P., \& Davis, M. 1998, ApJ, 500, 525
Storm, J., Gieren, W., Fouqué, P., et al. 2011, A\&A, 534, A95

Tammann, G. A., Sandage, A., \& Reindl, B. 2003, A\&A, 404, 423

Tonry, J., \& Schneider, D. P. 1988, AJ, 96, 807

Tonry, J. L., Blakeslee, J. P., Ajhar, E. A., \& Dressler, A. 2000, ApJ, 530, 625

Toomer, G. 1974, Archives for the History of the Exact Sciences, 14, 126

Tully, R. B., \& Courtois, H. M. 2012, ApJ, 749, 78

Tully, R. B., \& Fisher, J. R. 1977, A\&A, 54, 661

Tully, R. B., \& Pierce, M. J. 2000, ApJ, 533, 744

Tully, R. B., Shaya, E. J., Karachentsev, I. D., et al. 2008, ApJ, 676, 184

Tully, R. B., Rizzi, L., Shaya, E. J., et al. 2009, AJ, 138, 323

Tully, R. B., Courtois, H. M., Dolphin, A. E., et al. 2013, AJ, 146, 86

Udalski, A., Szymanski, M., Kubiak, M., et al. 1999, Acta Astron., 49, 201

Véron-Cetty, M.-P., \& Véron, P. 2010, A\&A, 518, A10 